\title{
Surrogate Modeling Based Cognitive Decision Engine for Optimization of WLAN Performance
}

\author{
David Plets ${ }^{\mathrm{a}}$, Krishnan Chemmangat ${ }^{\mathrm{b}}$, Dirk Deschrijver ${ }^{\mathrm{a}}$, Michael Mehari ${ }^{\mathrm{a}}$, Selvakumar Ulaganathan ${ }^{\mathrm{a}}$, \\ Mostafa Pakparvar, Tom Dhaene ${ }^{\mathrm{a}}$, Jeroen Hoebeke ${ }^{\mathrm{a}}$, Ingrid Moerman ${ }^{\mathrm{a}}$, Emmeric Tanghe $^{\mathrm{a}}$ \\ ${ }^{a}$ Ghent University/iMinds, Gaston Crommenlaan 8, Bus 201, 9050 Gent, Belgium \\ ${ }^{b}$ Department of Electrical and Electronics Engineering, National Institute of Technology Karnataka (NITK) - Surathkal, \\ Mangalore - 575025, India
}

\begin{abstract}
Due to the rapid growth of wireless networks and the dearth of the electromagnetic spectrum, more interference is imposed to the wireless terminals which constrains their performance. In order to mitigate such performance degradation, this paper proposes a novel experimentally verified surrogate model based cognitive decision engine which aims at performance optimization of IEEE 802.11 links. The surrogate model takes the current state and configuration of the network as input and makes a prediction of the QoS parameter that would assist the decision engine to steer the network towards the optimal configuration. The decision engine was applied in two realistic interference scenarios where in both cases, utilization of the cognitive decision engine significantly outperformed the case where the decision engine was not deployed.
\end{abstract}

Keywords: Cognitive Decision Engine, Surrogate Modeling, Interference Management, Dynamic Spectrum Access, WiFi, WLAN

\section{Introduction}

With the increasing demand of the scarce electromagnetic spectrum and rising interference effects, cognitive radios (CR) have become a promising solution to address spectrum over-utilization. To this end, cognitive solutions are of paramount importance in wireless networks to ensure that their performance is not degraded by the external interference, ensuring continuous connectivity and providing a good Quality of Service (QoS). A well-established component that targets optimal network communication is the Cognitive Decision Engine (CDE). The CDE is an intelligent module that aims to optimize network per-

\footnotetext{
* Please address correspondence to

Email address: david.plets@intec.ugent.be (David Plets)
}

formance objectives (QoS) by making informed decisions upon changes in the wireless environment.

Three important aspects of a CDE comprise: (1) observation, (2) reconfiguration or adaptability and, (3) cognition which includes awareness, reasoning and learning [1]. In literature, these three characteristics have been addressed using various approaches, e.g. rule-based [2], case-based [3, 4, 5], search-based [6], knowledge-based [4] reasoning systems and many others. Such methods often require a certain set of representative cases or domain expert knowledge [7] to derive important analytical formulas or rules that steer the decision making process. The reader is referred to [1] for a detailed survey.

In this paper, a real-time CDE is developed that is based on surrogate modeling of the QoS. Moreover, the surrogate model is built dynamically, unlike in the machine learning approach where the models 
are static. Further, the CDE performance is experimentally tested. Specifically, the developed surrogate model-based CDE reconfigures the transmission parameters of a wireless LAN system in response to measured changes in the environment, with the goal to maximize the QoS at all times. The experimental demonstration and validation is done via two realistic use cases in a wireless testbed where the proposed algorithm is shown to optimize the performance of wireless links by changing various transmission parameters of the wireless terminals using surrogate model predictions. To the authors' knowledge, this paper is the first to describe, develop, and experimentally test such dynamical surrogate model-based CDE.

This paper is organized as follows: after reviewing the current literature in Section 2, Section 3 describes the CDE. This includes a description of the architecture and a discussion on the two main components: the surrogate model and the optimization algorithm. In Section 4, two real-life examples are provided in which experiments are performed in a wireless testbed to show the applicability and performance of the proposed CDE. Section 5 concludes this article.

\section{Related works}

In the context of cognitive frameworks that address efficient interoperability of homo/heterogeneous wireless networks, spectrum monitoring and decision making are the central topics addressed by the authors in the current literature. Radio environment maps (REMs) [8] play a key role for environment monitoring in many cognitive radio solutions. REMs represent an integrated database providing information such as spectrum availability, regulations, and also the degree of channel utilization [8].

The decision making mechanisms in the present literature exploit a multitude of Artificial Intelligence (AI) algorithms to derive proper decisions for operation of the cognitive wireless network. Artificial Neural Networks (ANN) have been used for radio parameter adaptation in CR $[9,10]$. The ANN determines radio parameters for given channel states with three optimization goals, including meeting the bit error rate (BER), maximizing the throughput and minimizing the transmit power. In [11], it is proposed to use the ANN to characterize the real-time achievable communication performance in CR. Since the characterization is based on runtime measurements, it provides a certain learning capability that can be exploited by the cognitive engine. The simulation results demonstrate good modeling accuracy and flexibility in various applications and scenarios. Moreover, in [12], the authors propose two ANN-based learning schemes which aim at optimizing the end user's data rate in dynamic environments by changing certain input parameters such as the radio access technology and its corresponding frequency. The two schemes are validated by two (unseen) data sets which are different to the two (seen) data sets which were used in the learning phase. The validation results shows that the ANN-based learning schemes pay off in maintaining the data rate of the end user in a cognitive radio network.

Game theory techniques have also been widely used in the context of cognitive radios. In [13], population game theory has been applied to model the spectrum access problem and develop distributed spectrum access policies based on imitation, a behavior rule widely applied in human societies consisting of imitating successful behaviors. In [14], the authors study the spectrum access problem in cognitive radio networks from a game-theoretical perspective. The problem is modeled as a non-cooperative spectrum access game where secondary users simultaneously access multiple spectrum bands left available by primary users, optimizing their objective function which takes into account the congestion level observed on the available spectrum bands.

Apart from all aforementioned AI methods, there are also methods in literature that are based upon ranking the channels in order of their capacity. The ranking is done by characterizing the channel activities and making estimations of the capacity accordingly. In particular, the authors in [15] propose a spectrum decision framework for cognitive radio networks which addresses QoS management of the secondary users in response to certain events such as appearance of a primary user or degradation of the QoS. Thus their proposed framework not only accounts for 
consideration of primary users, but also maintains the QoS delivered to the secondary users by making spectrum decision according to the channel activities. The current paper is therefore a logical continuance and extension of the work on (ANN) model based learning schemes [12] where we share a common architecture with [15] in tackling interference and optimizing the QoS. We extend the concept by bringing cognitive radio techniques for QoS management to the Industrial Scientific Medical (ISM) band WLANs as well as incorporating REMs and surrogate modeling based decision making to the framework.

\section{Cognitive Decision Engine}

\subsection{Architecture}

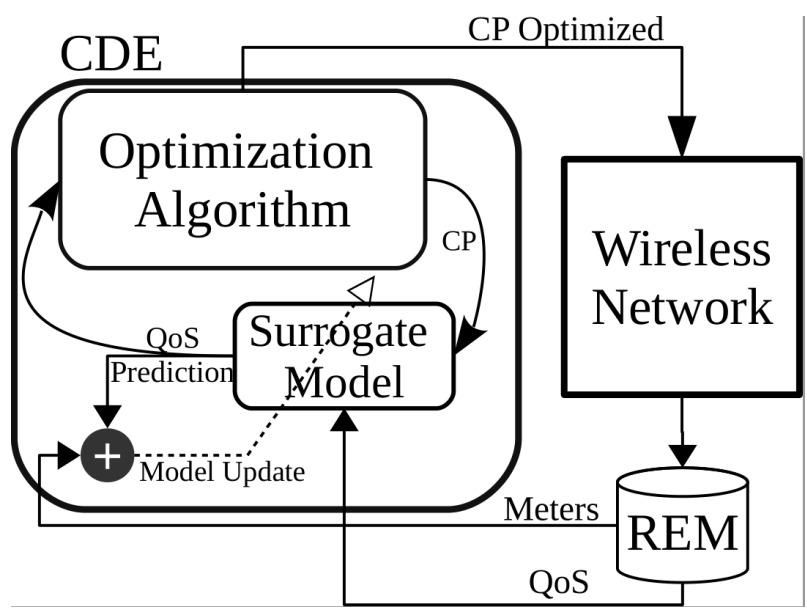

Figure 1: A high-level schematic of the cognitive decision strategy

A schematic overview of the decision engine is shown in Figure 1. The System under test is the wireless system that needs to be optimized in a dynamically changing environment. The Radio Environment Map (REM) is a map that continuously aggregates all information that is monitored from the environment through spectrum sensing, network sniffing, and benchmarking tests [16]. Based on the REM map, a set of meters or measurable parameters
(MP) can be extracted that allow the CDE to identify the current state of the environment. The Cognitive decision engine (CDE) is a self-learning decision engine that uses a surrogate model to optimize the QoS by defining suitable cognitive actions in response to the MP parameters that are derived from REM. These actions are enforced by tuning of a set of knobs or control/transmission parameters (CP) that specify the configuration of the wireless system. By storing the outcome of each action, the algorithm collects data samples that quantify the QoS behavior of the wireless system. The surrogate model is an analytical black- box model that characterizes and approximates this behavior. It is an input-output mathematical function that models the relation between the MP and CP parameters as inputs on one hand, and the resulting QoS performance of the wireless system that would be observed as an output on the other hand. Rather than building a static set of models upfront, a self-learning modeling strategy is thus adopted, limiting the set of a priori experiments. The outcome of the actions that were executed by the optimization process are used in an online feedback loop to update/improve the accuracy of surrogate model during its operation (online). The predictive surrogate model generalizes the obtained knowledge towards environmental conditions and configurations that are different from those that were observed during training by means of interpolation. This system allows a real-time optimization of the QoS performance by tuning the CP parameters of the wireless system according to the MP parameters that are obtained from the REM. A more detailed explanation of these components will be provided in the later sections.

\subsection{Surrogate Model}

\subsubsection{Notational conventions}

In order to model the QoS performance of the wireless system, a set of data samples must be collected by performing a limited set of $k=1, \ldots, K$ experiments on a wireless testbed (w-iLab.t) as described in Section 4. Each experiment $k$ is defined by a vector of $i=1, \ldots, n_{c}$ knobs spanning the control space $\mathbf{C}_{k}=\left\{C_{k}^{i}\right\}$, and a vector of $j=1, \ldots, n_{m}$ spanning the meter space $\mathbf{M}_{k}=\left\{M_{k}^{j}\right\}$. As mentioned 
in the previous section, the $\mathbf{C}_{k}$ space comprises the control parameters that must be configured by the decision engine, whereas the $\mathbf{M}_{k}$ space comprises a set of features that are extracted from the REM. The union of the control space $\mathrm{C}$ and the meter space $\mathrm{M}$ is called the parameter space. The outcome of each experiment $k$ corresponds to a resulting $Q o S_{k}$ performance value. Depending on the ultimate usage of the wireless network, the target QoS parameter may be throughput, delay, jitter, or a combinatorial metric such as audio or video quality. Each experiment $k$ is referred to as a data sample and will be represented by a tuple: $\left\{\mathbf{C}_{k}, \mathbf{M}_{k}, Q o S\left(\mathbf{C}_{k}, \mathbf{M}_{k}\right)\right\}$.

\subsubsection{Surrogate model type}

All experiments that are performed constitute a dataset that will be used to build a surrogate model $f$ such that $f\left(\mathbf{C}_{k}, \mathbf{M}_{k}\right)=Q o S_{k}$. As surrogate models must be built and updated in real time, it is important to select an interpolation strategy that is simple and fast. It should 1) not require a lot of model tweaking; 2) be able to build models that are sufficiently accurate; 3 ) be robust towards noise and imperfections in the data; 4) have acceptable running times. In literature, many modeling algorithms are described, such as e.g. kriging [17], artificial neural networks [18, 19], radial basis function [20], etc. Most of the time, they require a trial-and-error approach for selecting a suitable set of hyperparameters (e.g., the number of neurons, the number of hidden layers, the model complexity, etc.). Also the model selection and cross-validation process can be very time consuming. With these considerations in mind, it is preferable to consider local interpolation schemes such as multi-linear interpolation [21], tesselationbased simplicial interpolation [22] and Shepard's interpolation $[23,24]$. The Shepard interpolation algorithm was found to be the most adequate approach because of the simplicity of the underlying principle, and the speed in calculation. By using Shepard's interpolation, a model is built that exactly predicts the QoS performance for experiments that were performed previously, and approximates the QoS for experiments that have not yet been performed (i.e. different values of $\mathbf{C}$ and $\mathbf{M}$ ), based on a distance-based similarity measure and an appropriate normalization of the parameter space.

\subsubsection{Model Building}

In order to build the surrogate model, a representative set of data samples must be collected to build a model having sufficient accuracy to make reliable predictions of the QoS. Data samples are collected in such a way that the model accuracy can be maximized while minimizing the number of experiments needed. This happens in an online training phase that is executed prior to the deployment of the decision engine. In sequential steps, a well-chosen set of experiments are performed by making a balanced trade-off between two different criteria, namely exploration and exploitation.

Exploration phase. In the exploration phase, different settings of the knobs $\mathbf{C}$ are explored in order to cover the parameter space as evenly as possible. The idea is that the most informative experiments are those that are as different as possible from those that were performed previously. Thereto, a distancebased exploration criterion is used to let the dataset grow over time. First, a large set of candidate settings is generated using a Monte Carlo method for the knobs in such a way that they cover the entire control space. For all the candidate settings of the knobs $\mathbf{C}$, the distance of each candidate point to all other data samples in the set is calculated and the farthest point is selected. So, if an $n_{c}$-dimensional control space with $d=1, \ldots, D$ discrete candidate settings for the knobs is considered, then the proposed setting of the knobs is the one that maximizes the minimum distance to the available data samples $k=1, \ldots, K$.

$$
d^{\text {best }}=\underset{d}{\operatorname{argmax}}\left[\min _{k} h_{k}^{d}\right]
$$

where $h_{k}^{d}$ represents the Euclidean distance between data sample $k$ in the dataset and candidate setting $d$. The values of the meters are set to those at current time $\mathbf{M}=\mathbf{M}_{\text {current }}$.

$$
h_{k}^{d}=\sqrt{\sum_{i=1}^{n_{c}}\left(C_{d}^{i}-C_{k}^{i}\right)^{2}+\sum_{j=1}^{n_{m}}\left(M_{\text {current }}^{j}-M_{k}^{j}\right)^{2}}
$$


Once this exploratory setting $d^{\text {best }}$ of the knobs is applied on the system under test, the resulting QoS is evaluated and the outcome is added as an additional data sample to the dataset. As the environment changes over time, the meters $\mathbf{M}_{\text {current }}$ will change dynamically over time and sequentially data samples are added to the dataset. This leads to more accurate predictions of the surrogate model built from it.

Exploitation phase. As the algorithm collects more data samples, it can also exploit data from previous experiments to make the model more accurate in those regions where the optimum configurations (i.e. those with higher QoS values) are located. To this end, the output of the intermediate surrogate models can be used to identify these settings. Just like in the exploration phase, the meters are monitored and a large set of candidate settings for the knobs are generated using Monte Carlo in such a way that they cover the entire control space. For all discrete candidate settings $d$ of the knobs $\mathbf{C}$, the surrogate model is evaluated and the setting $\mathbf{C}^{\text {opt }}$ is chosen for which the Shepard's model predicts the highest $P Q o S=f\left(\mathbf{C}_{d}, \mathbf{M}_{\text {current }}\right)$ value.

$$
\mathbf{C}^{\text {opt }}=\underset{d}{\operatorname{argmax}} f\left(\mathbf{C}_{d}, \mathbf{M}_{\text {current }}\right)
$$

The values of meters $\mathbf{M}_{\text {current }}$ in (3) are again set to those at the current time. Once the optimal setting $\mathbf{C}^{\text {opt }}$ of the knobs is applied on the system, the resulting $Q o S\left(\mathbf{C}^{\text {opt }}, \mathbf{M}_{\text {current }}\right)$ value is evaluated and the outcome is compared to the predicted $P Q \circ S=$ $f\left(\mathbf{C}^{\text {opt }}, \mathbf{M}_{\text {current }}\right)$ of the surrogate model.

$$
\left|f\left(\mathbf{C}^{\text {opt }}, \mathbf{M}_{\text {current }}\right)-Q o S\left(\mathbf{C}^{\text {opt }}, \mathbf{M}_{\text {current }}\right)\right|<\tau
$$

If the discrepancy of the outcome is larger than a predefined threshold $\tau$, then the prediction of the model was not sufficiently accurate and a corresponding data sample $\left\{\mathbf{C}^{\text {opt }}, \mathbf{M}_{\text {current }}, Q o S\left(\mathbf{C}^{\text {opt }}, \mathbf{M}_{\text {current }}\right)\right\}$ is added to the dataset. If the surrogate model prediction was sufficiently accurate, then the experiment can be discarded. This step improves the accuracy of the surrogate model in regions where optima are located.

\subsection{Optimization algorithm}

Once a surrogate model is considered to be sufficiently accurate, it can be deployed in the cognitive decision framework for real-time optimization. The optimization algorithm (see Fig. 1) continuously monitors the QoS performance and the values of $\mathbf{M}$ that are changing dynamically over time. If a network performance degradation is detected, a genetic algorithm can be used to solve (3) and to determine the optimum value $\mathbf{C}^{\text {opt }}$ of the knobs [25]. For usecases with a relatively small number of parameters, one can generate a large set of candidate settings for the knobs using Monte Carlo in such a way that they cover the entire control space, from which the optimum configuration can be chosen. This takes only a fraction of a second because the Shepard model is an analytical function that is fast to evaluate. The use of genetic algorithms is a possible way to economize on the number of function evaluations and to speedup the optimization in the case of complex networks with many parameters. In cases where the result of the optimization step was unsuccessful in predicting the QoS, the outcome of the experiment can be added as additional data to improve the model predictions in an online fashion.

\section{Experimental Examples: Practical Case studies}

\subsection{Setup configuration}

All practical case studies are conducted in a pseudo-shielded testbed environment w-iLab.t [26] in Ghent, Belgium. The nodes in the testbed are mounted in an open room $(66 \mathrm{~m} \mathrm{x} 20.5 \mathrm{~m})$ in a grid configuration with an x-separation of $6 \mathrm{~m}$ and a $\mathrm{y}$ separation of $3.6 \mathrm{~m}$. Figure 2 shows the ground plan of the test lab with an indication of the location of the nodes. Each node has two Wi-Fi interfaces (Sparklan WPEA-110N/E/11n mini PCIe 2T2R chipset: AR9280). Furthermore, a ZigBee sensor node and a USB 2.0 Bluetooth interface (Micro CI2v3.0 EDR) are incorporated into each node.

The CDE is implemented in MATLAB and runs on a separate computer. It writes settings for controlor transmission parameters into an SQL database. A 
Table 1: Mean Opinion Score.

\begin{tabular}{|c|l|l|}
\hline MOS & Quality & Impairment \\
\hline 1 & bad & very annoying \\
2 & poor & annoying \\
3 & fair & slightly annoying \\
4 & good & perceptible, not annoying \\
5 & excellent & imperceptible \\
\hline
\end{tabular}

Java program then continuously polls the database for input, transforms the command into an OMF script which is executed as an experiment on the testbed and writes the outcome (i.e., a QoS metric) back into the SQL database which can be queried by the CDE. Hence, the main overhead is computational power on the computer that runs the CDE.

Depending on the ultimate usage of the wireless network, the target QoS parameter may be throughput, delay, jitter, or a combinatorial metric such as audio or video quality. This surrogate modelling approach requires a target metric that changes continuously and can be modeled by an analytical function. The conflicting nature of different QoS parameters influencing the audio/video quality such as throughput and latency [27], requires using scalar metrics such as the audio Mean Opinion Score (MOS) [28] which is represented by a scalar value in between 1 and 5 (see Table 1). For a given audio stream, the MOS value is easily calculated by feeding the ITU G107 E model [28] with the values of throughput and jitter. Note that, since MOS scores are bounded in between 1 to 5 , a value of $\tau=0.5$ is proposed in this paper.

For each experiment that is configured, the sender node will stream an audio file to its receiver(s) over a short period of time. At the same time, external interference is generated by a collocated pair of IEEE802.11 compliant nodes by means of iperf [29] data generator. We refer to these two nodes as the interference generating group, abbreviated as INT. These nodes generate a continuous interference traffic on a different channel. The effect of this external interference on the QoS performance of the wireless system is highly unpredictable in nature, so this experiment can be seen as an analogy to real-life oper- ation of the system.

\subsection{Scenario I: Single-link optimization of MOS}

The setup that was considered is a typical scenario of two IEEE 802.11 standard compliant nodes, operating in infrastructure mode with $802.11 \mathrm{~g}$ standard and generating traffic on down-link. We refer to these two nodes as the System Under Test (SUT). In this scenario, the sender of the SUT is located at node 48 and the location of the receiver node of the SUT (knob 1) can be changed over a fixed range of discrete positions, varying from node 56 to 60 . In addition to this parameter, the transmit power $\left(\mathrm{P}_{\mathrm{Tx}}\right)$ of the sender node of the SUT (knob 2) can also be varied in-between 1 and $20 \mathrm{dBm}$. The aim of the CDE is to tune these 2 parameters such that the MOS over the corresponding link is maximized over the period of audio streaming between the SUT sender and receiver. Each time step is set to 12 seconds which is the duration of a normal English sentence audio file that is streamed over the link.

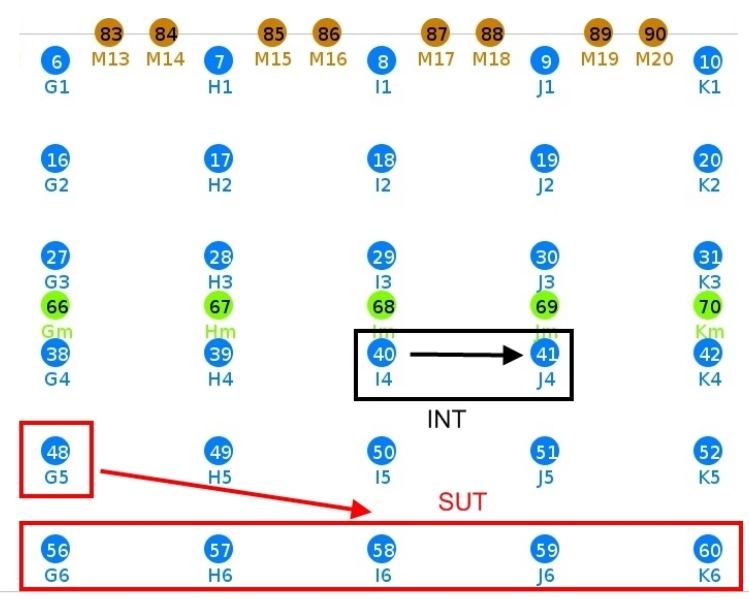

Figure 3: Layout of testbed for Scenario I.

In addition to the SUT, the sender node and the receiver node of the interference generating group (INT) are located at nodes 40 and 41, respectively. As mentioned earlier, these nodes generate a continuous amount of interference on channel 12, which is 


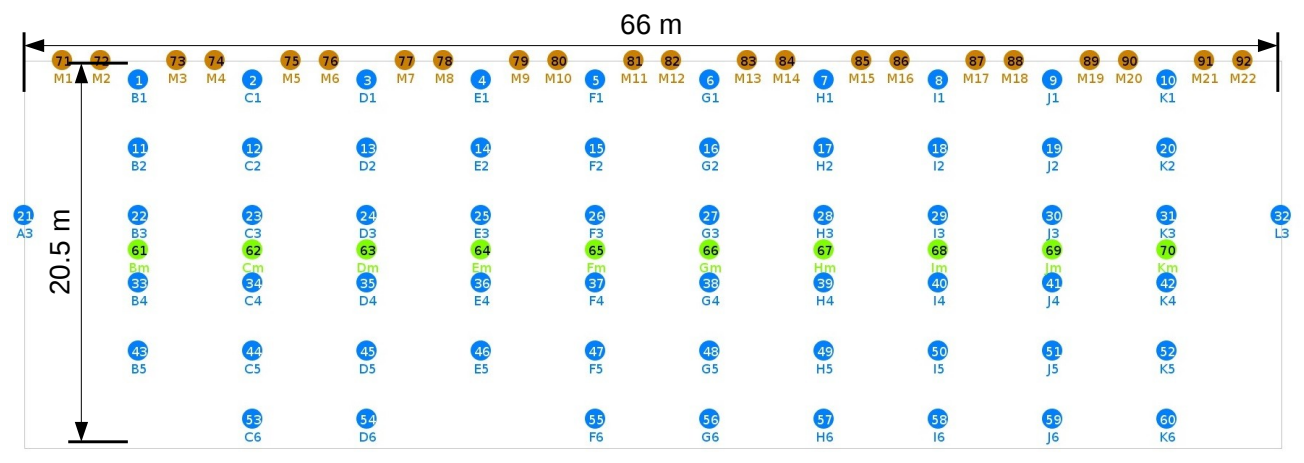

Figure 2: Layout of the W-iLab.t living lab test environment $(66 \mathrm{~m} \times 20.5 \mathrm{~m})$ with indication of the node numbers.

nearby the channel 10 of the SUT. Previous experimental results in [30] have shown that INT is more likely to negatively impact the QoS on the SUT link if they are operating on overlapping channels (due to the malfunctioning of the Carrier Sense Multiple Access/ Collision Avoidance (CSMA/CA) mechanisms of the IEEE 802.11 terminals on overlapping channels). The layout of the testbed for this scenario is visualized in Figure 3.

In addition to the knobs, also some meters must be derived to assess the current state of the environment. To this end, RSSI (Received Signal Strength Indication) information of INT at the SUT sender can be derived from the REM. This meter provides additional information about the interference pattern. Hence, the CDE should monitor those values and optimize both the location of the receiver as well as the Tx power of the sender of the SUT accordingly, in such a way that the MOS score is maximized. Table 2 lists the parameters that are considered during the cognitive decision making.

\subsubsection{Model building}

Since a real-time response of the CDE is desired, the engine is first subjected to a learning phase where the surrogate model is built. During this phase, an initial set of experiments are performed to learn the resulting MOS score over the SUT link as a function of 3 parameters listed in Table 2 . In order to create a dynamic environment, the transmit power of the INT is switched from a low value (e.g. $5 \mathrm{dBm}$ ) to a higher value (e.g. $20 \mathrm{dBm}$ ) after a certain number of time steps. This creates an interference pattern that is changing over time. As such, it exposes the algorithm to different levels of interference. While the measured RSSI values are changing, multiple experiments are performed by changing the knobs in such a way that the parameter space is well covered, i.e. A balanced trade-off is made between exploration and exploitation of the design space. Inbetween the first and the last sample of the training set, the ratio between both criteria decreases (increases) linearly from $100 \%-0 \%$ to $0 \%-100 \%$ respectively. Alternative balancing schemes can also be used as described in [31]. The 45 experiments that were performed during model building are shown in Figure 4.

Using these selected samples, an interpolation model is built by the CDE with the aim of making MOS score predictions over the parameter space. The prediction of this model can guide the switching between different wireless configurations over time in order to maximize the MOS score.

\subsubsection{Model deployment}

A hold-out set of 10 experiments is used to validate the accuracy of the surrogate model, as shown in Figure 5. In the ideal case, all data samples (marked by a red dot) are located close to the linear solid line which indicates a perfect agreement. Taking into account stochastic variability of the testbed, it is found that all experiments lie with a narrow boundary (marked 
Table 2: Overview of parameter list considered for Scenario I.

\begin{tabular}{|c|c|c|c|c|}
\hline Sl. No. & Type & Name & Values & Unit \\
\hline 1 & Knob & Position Rx node for SUT & {$[56,57,58,59,60]$} & - \\
2 & Knob & Tx Power of Sender SUT & {$[1,2, \ldots, 20]$} & $\mathrm{dBm}$ \\
3 & Meter & Interference RSSI at SUT sender & - & $\mathrm{dBm}$ \\
\hline
\end{tabular}

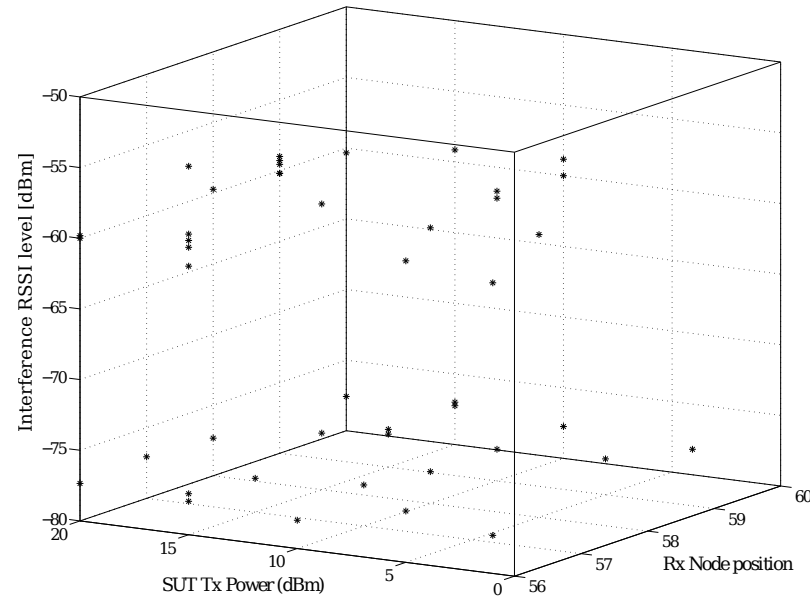

Figure 4: Scenario I: Experiments performed during the learning phase of the CDE. Each experiment is represented by a dot.

by 2 linear dashed lines), that corresponds closely to the model accuracy threshold $\tau$ as chosen in (4).

Figure 6 shows the predicted MOS score of the surrogate model when the SUT receiver node is fixed at node 58 , while evaluating the model for any possible value of the interference RSSI (meter) and Tx power of the SUT sender (knob), see vertical slice in Figure 7. As expected, it is seen that a better MOS can be obtained for lower RSSI values from the external interference and higher values of the Tx power of SUT. Note that the model is only locally accurate (i.e. it is trained within the operating ranges of the meters that were encountered during model building to make the algorithm scalable), however the interpolation routine aims to generalize this to other interference levels.

In order to assess the performance of the CDE

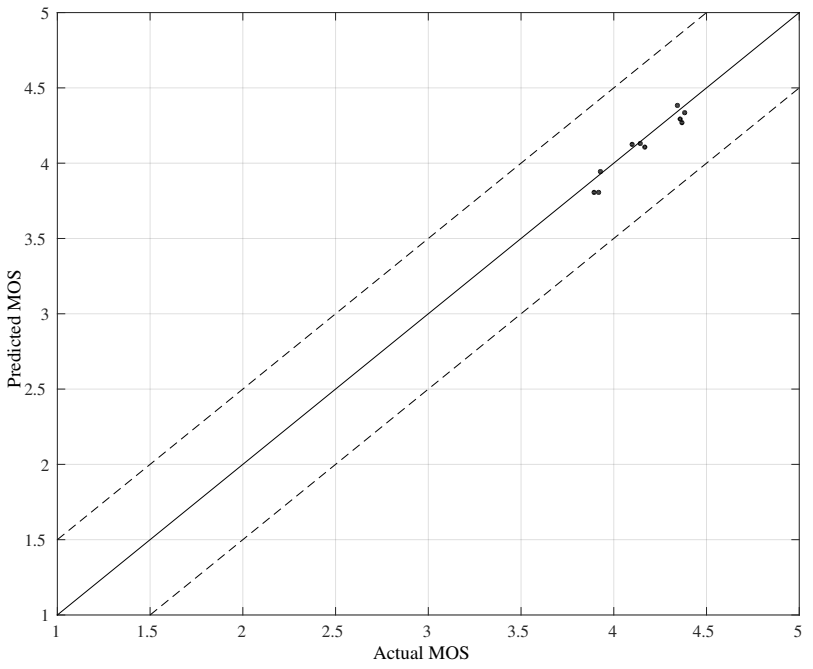

Figure 5: Scenario I: Actual MOS versus predicted MOS for 10 experiments. 


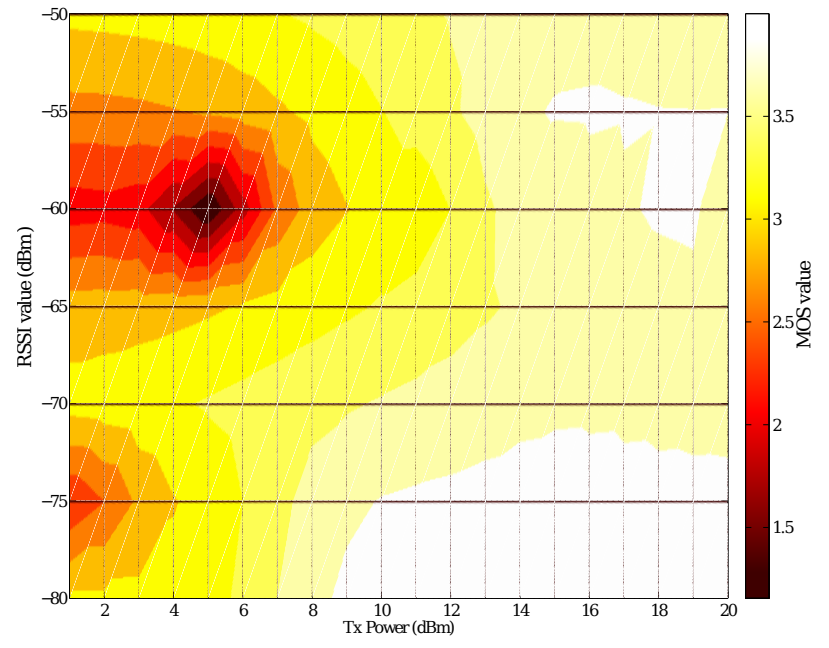

Figure 6: Scenario I: 2D surrogate model response for fixed Rx node $=58$.

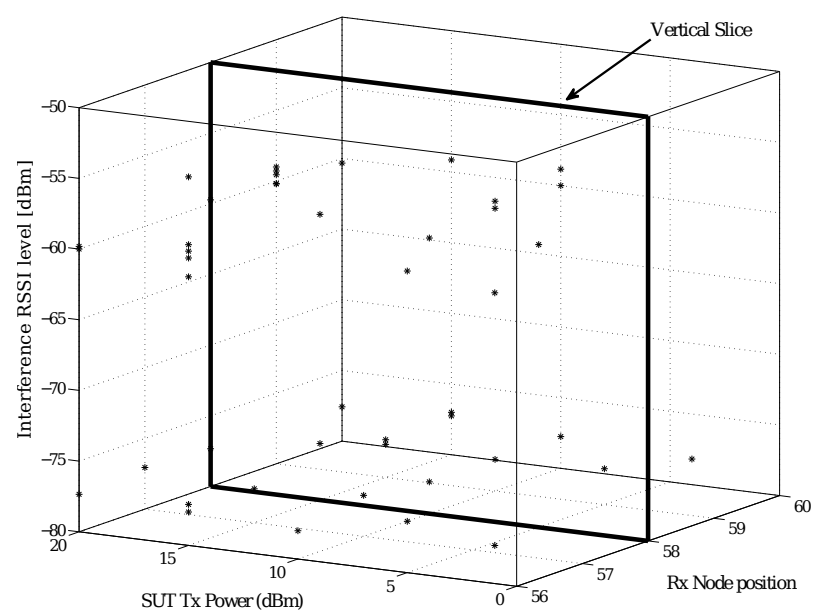

Figure 7: Scenario I: 2D surrogate model for fixed $\mathrm{Rx}$ node $=$ 58.

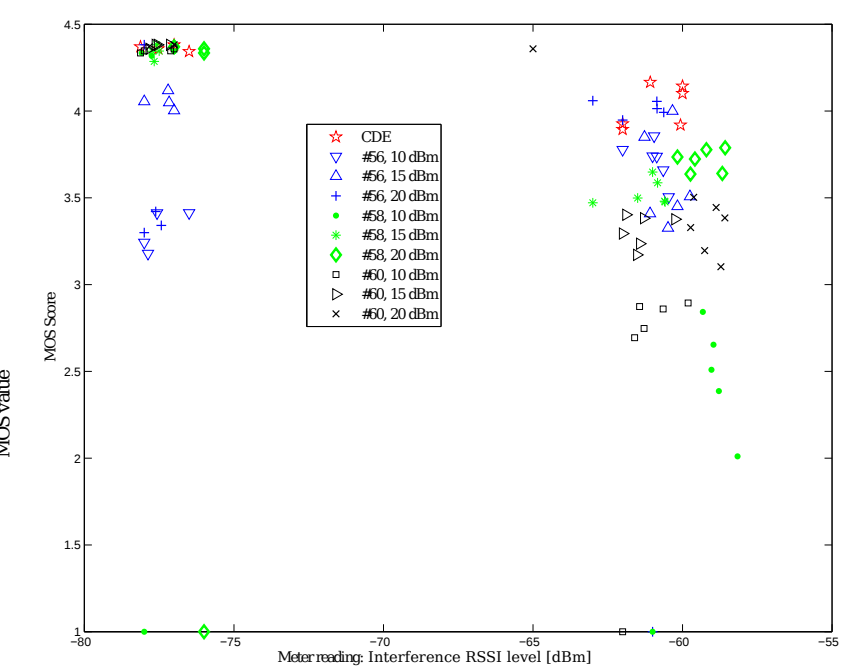

Figure 8: Scenario I: MOS score versus interference RSSI at SUT sender for static and CDE assisted cases.

in this scenario, the resulting MOS score over the SUT link is compared to several static cases where the knobs are set to a fixed configuration (no cognitive tuning). As an example, 10 different experiments were performed and the resulting MOS scores are visualized in Figure 8. This figure shows the obtained MOS score as a function of the measured RSSI value of INT at the SUT. Note that the RSSI values for each case are slightly different because this parameter is subject to stochastic variability. The RSSI is also not constant, because the interference pattern of INT changes over time. Recall that the sender of the INT switches its Tx power from a low value $(5 \mathrm{dBm})$ to a high value $(20 \mathrm{dBm})$ after 4 time steps in order to create a dynamically changing environment. When compared to several other static configurations, it is found that the CDE gives MOS score of 3.5 or higher (which is in-between the subjective rating of "fair" and "excellent"). If the same experiment is repeated without cognitive tuning, the QoS may degrade significantly (i.e., MOS score of 1 , which is "bad"). A more systematic quantification of the CDE performance is given in Table 3, where the resulting MOS score is averaged over the 10 experiments. It is shown that the cognitive solution can lead to an improve- 
Table 3: Performance evaluation of CDE for Scenario I. Imp. is an abbreviation for relative improvement.

\begin{tabular}{|c|c|c|}
\hline Experiment & Avg. MOS & Imp. (\%) \\
\hline CDE & 4.16 & \\
Node:56, Tx Power $=10$ & 3.55 & 17.12 \\
Node:56, Tx Power $=15$ & 3.77 & 10.14 \\
Node:56, Tx Power $=20$ & 3.55 & 17.14 \\
Node:58, Tx Power $=10$ & 2.74 & 51.84 \\
Node:58, Tx Power $=15$ & 3.85 & 8.00 \\
Node:58, Tx Power $=20$ & 3.64 & 14.41 \\
Node:60, Tx Power $=10$ & 3.25 & 28.19 \\
Node:60, Tx Power $=15$ & 3.74 & 11.34 \\
Node:60, Tx Power $=20$ & 3.41 & 22.12 \\
\hline
\end{tabular}

ment in-between $8.00 \%$ and $51.84 \%$ when compared to the static configurations. As expected, it is found that configurations with a low Tx power of the SUT sender and a large distance between sender and receiver of the SUT give more room for improvement than other configurations. Also, it is found that the cognitive solution (which adaptively tunes the knobs) performs better than any of the static configurations.

Finally, the bracketed values in Figure 9 comprise the values of knob 1 and knob 2 respectively as chosen by the CDE, which corresponds to the decisions taken during 10 deployment experiments. The MOS score is shown as a function of the measured RSSI value of INT at sender SUT for the different experiments. It is seen that, when the RSSI values are relatively high for the INT, the CDE switches to node 56, which is located close to the sender SUT. It is also observed that the CDE does not necessarily pick the highest possible transmit power for the SUT sender for good performance.

The resulting MOS score of the cognitive solution is also compared to the performance of a static configuration over time in Figure 10. For the static case, both Tx power of the SUT and the location of the $\mathrm{Rx}$ node are set to some middle values. In the case of low external interference, both configurations give acceptable results, however once the interference level is increased (after 4 time steps), the cognitive solution is able to enforce more optimal choices that lead to an

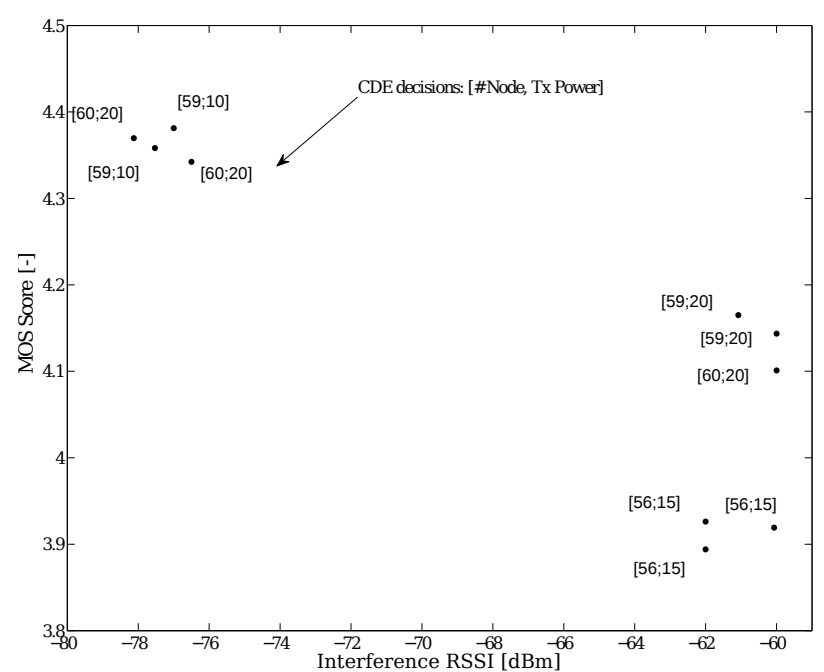

Figure 9: Scenario I: CDE decisions as a function of the meter reading (INT RSSI). Each decision is characterized with [Node number, Tx Power].

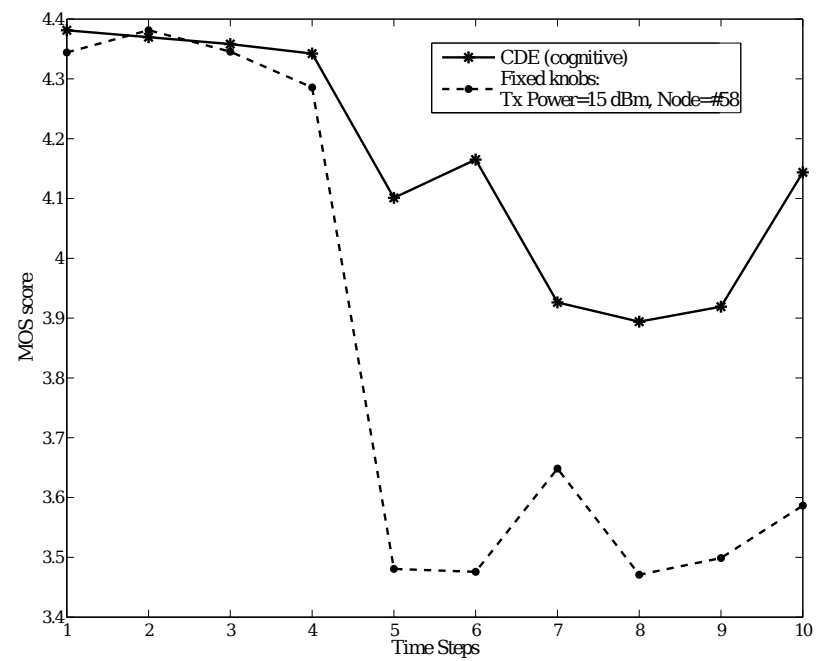

Figure 10: Scenario I: QoS performance of cognitive vs. static configuration. Each time step is 12 seconds. 
increase of the MOS. Hence, for high interference levels, the CDE is able to give significant improvements, compared to the static case.

\subsection{Scenario II: Multiple-link optimization of MOS}

This scenario describes a more complex case where 7 nodes are involved: 1 SUT sender (located at node 48 ) is surrounded by 4 SUT receivers (located at the nodes 37, 39, 55 and 57 respectively). Similarly as in the previous scenario, an INT sender-receiver pair (located at nodes 40-41) is again acting as an interferer on the SUT. A visualization of the network topology is provided in Figure 11.

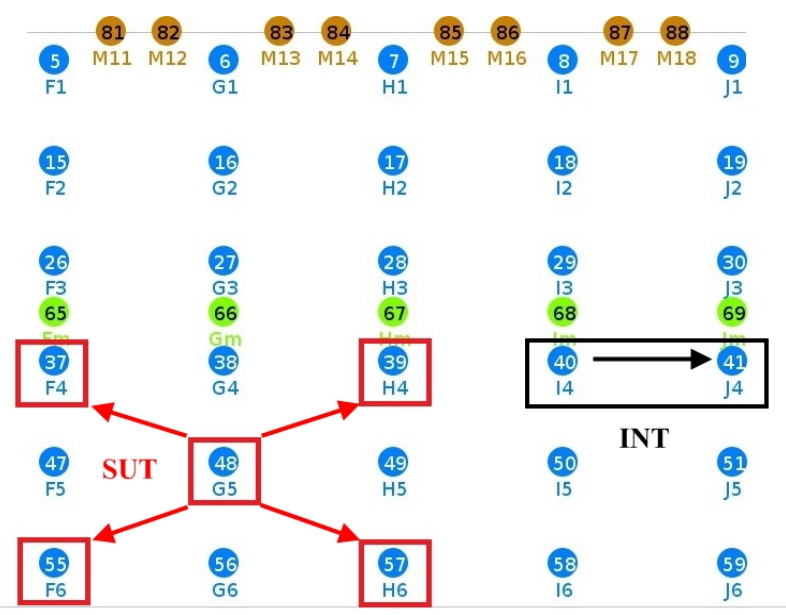

Figure 11: Layout of testbed for Scenario II.

\subsubsection{Building the surrogate model}

The aim of this experiment is to maximize the worst-case MOS score over all 4 SUT links simultaneously. The time step is again set to 12 seconds. Three parameters can be tuned by the CDE:

(i) Tx power of the SUT sender

(ii) Tx rate of the SUT sender

(iii) Frequency channel of the SUT

To this end, the following 2 meters must be monitored: (i) RSSI information at SUT sender

(ii) Channel information of the INT

An overview of the parameters is given in Table 4 . In order to create a dynamic environment, the channel of the INT is switched between different frequencies, leading to more abrupt (non-smooth) changes in the MOS score.

Similarly, for the model building phase of the algorithm 87 experiments were performed according to the exploration/exploitation trade-off in order to compute a surrogate model of the MOS score.

\subsubsection{Model deployment}

Using this model, the CDE can optimize the worstcase MOS score over all the links by enforcing certain actions. Figure 12 compares the predicted MOS score of the surrogate model to the actual MOS score obtained after executing some validation experiments and it is found that a very good agreement is again obtained.

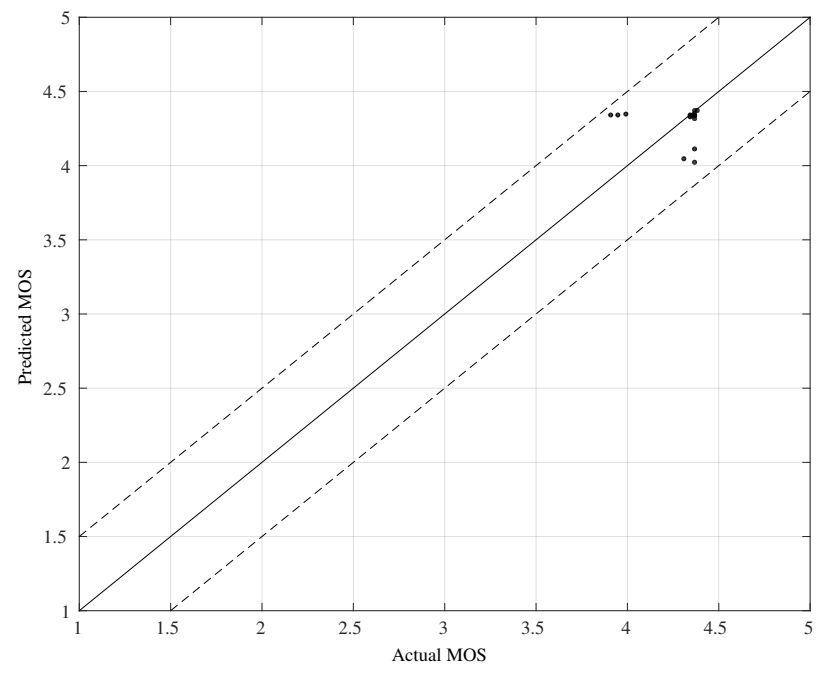

Figure 12: Scenario II: Actual MOS versus predicted MOS for 15 experiments.

Figure 13 visualizes the worst-case MOS score as a function of measured RSSI values at SUT in a dynamic environment where the interferer switches from 
Table 4: Overview of parameter list considered for Scenario II.

\begin{tabular}{|c|c|c|c|c|}
\hline Sl. No. & Type & Name & Values & Unit \\
\hline 1 & Knob & Tx Power of Sender SUT & {$[1,2, \ldots, 20]$} & $\mathrm{dBm}$ \\
2 & Knob & Tx Rate of Sender SUT & {$[1,2, \ldots, 11]$} & $\mathrm{Mbps}$ \\
3 & Knob & Frequency channel of SUT & {$[1,2, \ldots, 12]$} & - \\
4 & Meter & Interference RSSI at SUT sender & - & $\mathrm{dBm}$ \\
5 & Meter & Frequency channel of INT & - & - \\
\hline
\end{tabular}

Table 5: Performance evaluation of CDE for Scenario II.

\begin{tabular}{|c|c|c|}
\hline Experiment & Avg. worst-case MOS & Improvement (\%) \\
\hline with CDE & 4.28 & \\
no CDE, Channel=11, Tx Power=20, Tx Rate=11M & 3.69 & 15.99 \\
no CDE, Channel=4, Tx Power=20, Tx Rate=11M & 3.85 & 11.17 \\
\hline
\end{tabular}

channel 2 to 7 and then to 12 after a certain number of time steps. In the static configurations, the channel, Tx power and Tx rate of the SUT sender are set to fixed values. The CDE on the other hand, optimizes the parameters in a cognitive way. The worstcase MOS scores when averaged over 15 experiments are listed in Table 5. It is clear that the CDE yields a significant QoS performance gain when compared to other static solutions.

The resulting MOS score of the cognitive solution (CDE) is also compared to the performance of the static configurations in Figure 14.a. For the static case, both Tx power of the SUT and the location of the $\mathrm{Rx}$ node are set to the values listed in Table 5 . The channel of the INT is changed as shown in Figure 14.b. Hence, in a dynamic environment where the INT pattern changes, neither fixed configuration (Case 1 and Case 2 in the figure) turns out to be optimal in the general case. The CDE algorithm detects that the INT changes to a different channel, and is able to adjust the knobs of the SUT in such a way that a good MOS score $(>3.9)$ is preserved.

\section{Conclusion}

A novel approach for decision making is presented in the context of cognitive ISM band WLANs where

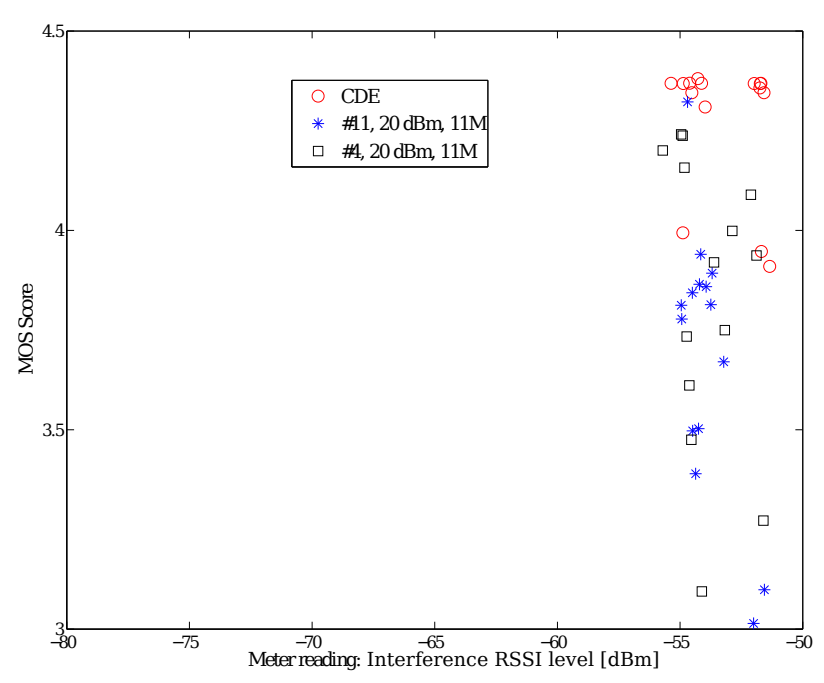

Figure 13: Scenario II: MOS score versus interference RSSI at SUT sender. 

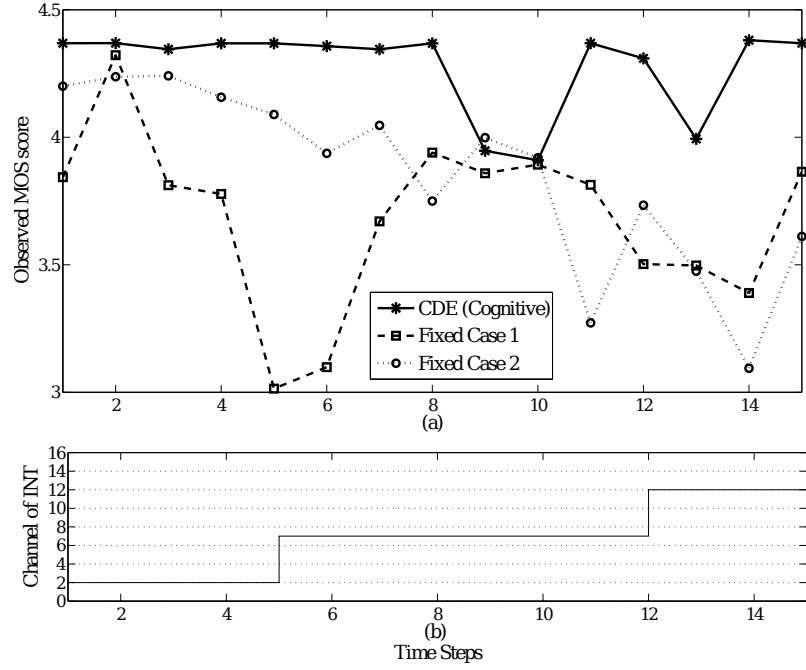

Figure 14: Scenario II: (a) QoS performance of cognitive vs. static configuration, (b) Channel of INT. each time step is 12 seconds.

all users have equal regulatory status in terms of spectrum utilization. The method incorporates surrogate models for predicting the performance of the network by monitoring environmental parameters and transmission parameters. It improves the models by following the accuracy of its predictions in real time. The generic design of the algorithm is beneficial for different scenarios with different set of parameters. As proof of concept, two use cases were experimentally investigated to verify the efficiency of this method in optimizing the performance of wireless networks. In the first use case, by changing the location of the receiver node, the average audio Mean Opinion Score (MOS) of an IEEE 802.11g compliant link was optimized by a factor of $30 \%$ compared to the same fixed configuration. In the second use case, by changing the transmission power, transmission rate and frequency channel of the sender in an audio conferencing scenario (with multicast traffic), the worst case MOS was improved by a factor of $50 \%$ compared to the static configuration.

In order to handle large networks with many parameters, the $\mathrm{CDE}$ can be extended with additional modules that contain expert rules or domain knowl- edge. This way, one can relax the assumption of a self-learning, black-box algorithm that no prior knowledge about the network behaviour is available. It is expected that this will significantly improve scalability of the approach and reduce the overall training times. Large scale implementation of the algorithm in different environments such as home, office, industry, etc. would therefore be the topic of future research. Finding the most appropriate set of meters and knobs in different scenarios on the one hand, and, coupling the model-based predictions with heuristic decision making methods on the other hand, would lead to useful endeavors aiming at improving the current algorithm.

\section{Acknowledgments}

The research activities that have been described in this paper were funded by Ghent University, iMinds, the Fund for Scientific Research in Flanders (FWOV) and the Interuniversity Attraction Poles Programme BESTCOM initiated by the Belgian Science Policy Office. This paper is also the result of research carried out as part of the QoCON project funded by iMinds. QoCON is being carried out by a consortium of the industrial partners: Televic, Option and Barco in cooperation with iMinds research groups: IBCN (UGent), WiCa (UGent), SMIT (VUB), PATS (UA) and IMEC.

[1] A. He, K. K. Bae, T. R. Newman, J. Gaeddert, K. Kim, R. Menon, L. Morales-Tirado, J. J. Neel, Y. Zhao, J. H. Reed, et al., A survey of artificial intelligence for cognitive radios, Vehicular Technology, IEEE Transactions on 59 (4) (2010) 1578-1592.

[2] B. G. Buchanan, E. H. Shortliffe, et al., Rulebased expert systems, Vol. 3, Addison-Wesley Reading, MA, 1984.

[3] D. B. Leake, Case-based reasoning, The knowledge engineering review 9 (01) (1994) 61-64.

[4] Y. Zhao, J. Gaeddert, L. Morales, K. Bae, J.-S. Um, J. Reed, Development of radio environment map enabled case- and knowledgebased learning algorithms for ieee 802.22 
wran cognitive engines, in: Cognitive Radio Oriented Wireless Networks and Communications, 2007. CrownCom 2007. 2nd International Conference on, 2007, pp. 44-49. doi:10.1109/CROWNCOM.2007.4549770.

[5] A. He, J. Gaeddert, K. K. Bae, T. R. Newman, J. H. Reed, L. Morales, C.-H. Park, Development of a case-based reasoning cognitive engine for ieee 802.22 wran applications, SIGMOBILE Mob. Comput. Commun. Rev. 13 (2) (2009) 3748. doi:10.1145/1621076.1621081.

[6] J. D. Ser, M. Matinmikko, S. Gil-Lpez, M. Mustonen, Centralized and distributed spectrum channel assignment in cognitive wireless networks: A harmony search approach, Applied Soft Computing 12 (2) (2012) 921 - 930. doi:http://dx.doi.org/10.1016/j.asoc.2011.10.001.

[7] M. Pakparvar, D. Plets, E. Tanghe, D. Deschrijver, W. Liu, K. Chemmangat, I. Moerman, T. Dhaene, L. Martens, W. Joseph, A cognitive QoS management framework for WLANs, EURASIP Journal on Wireless Communications and Networking 2014 (1) (2014) 191.

[8] Y. Zhao, L. Morales, J. Gaeddert, K. Bae, J.S. Um, J. Reed, Applying radio environment maps to cognitive wireless regional area networks, in: New Frontiers in Dynamic Spectrum Access Networks, 2007. DySPAN 2007. 2nd IEEE International Symposium on, 2007, pp. 115-118. doi:10.1109/DYSPAN.2007.22.

[9] M. Hasegawa, H.-N. Tran, G. Miyamoto, Y. Murata, S. Kato, Distributed optimization based on neurodynamics for cognitive wireless clouds, in: IEEE 18th International Symposium on Personal, Indoor and Mobile Radio Communications, 2007. PIMRC 2007, 2007, pp. 1-5. doi:10.1109/PIMRC.2007.4394658.

[10] Z. Zhang, X. Xie, Intelligent cognitive radio: Research on learning and evaluation of CR based on neural network, in: ITI 5th International Conference on Information and Communications
Technology, 2007. ICICT 2007, 2007, pp. 33-37. doi:10.1109/ITICT.2007.4475612.

[11] N. Baldo, M. Zorzi, Learning and adaptation in cognitive radios using neural networks, in: Consumer Communications and Networking Conference, 2008. CCNC 2008. 5th IEEE, 2008, pp. 998-1003. doi:10.1109/ccnc08.2007.229.

[12] K. Tsagkaris, A. Katidiotis, P. Demestichas, Neural network-based learning schemes for cognitive radio systems, Computer Communications 31 (14) (2008) 3394-3404.

[13] S. Iellamo, L. Chen, M. Coupechoux, Proportional and double imitation rules for spectrum access in cognitive radio networks, Computer Networks 57 (8) (2013) 1863 - 1879.

[14] J. Elias, F. Martignon, A. Capone, E. Altman, Non-cooperative spectrum access in cognitive radio networks: A game theoretical model, Computer Networks 55 (17) (2011) 3832 - 3846 .

[15] W.-Y. Lee, I. Akyldiz, A spectrum decision framework for cognitive radio networks, Mobile Computing, IEEE Transactions on 10 (2) (2011) 161-174. doi:10.1109/TMC.2010.147.

[16] Y. Zhao, Enabling cognitive radios through radio environment maps, Ph.D. thesis, Virginia Polytechnic Institute and State University (2007).

[17] J. Sacks, W. J. Welch, T. J. Mitchell, H. P. Wynn, Design and analysis of computer experiments, Statistical science 4 (4) (1989) 409-423.

[18] S. S. Haykin, Neural networks: a comprehensive foundation, Prentice Hall Englewood Cliffs, NJ, 2007.

[19] M. Hasegawa, H. N. Tran, G. Miyamoto, Y. Murata, S. Kato, Distributed optimization based on neurodynamics for cognitive wireless clouds, in: IEEE 18th International Symposium on Personal, Indoor and Mobile Radio Communications, 2007. PIMRC 2007., IEEE, 2007, pp. 1-5. 
[20] M. D. Buhmann, Radial basis functions: theory and implementations, Vol. 12, Cambridge university press, 2003.

[21] E. W. Cheney, Multivariate approximation theory: Selected topics, SIAM, 1986.

[22] C. B. Barber, D. P. Dobkin, H. Huhdanpaa, The quickhull algorithm for convex hulls, ACM Transactions on Mathematical Software (TOMS) 22 (4) (1996) 469-483.

[23] D. Shepard, A two-dimensional interpolation function for irregularly-spaced data, in: Proceedings of the 1968 23rd ACM national conference, ACM, 1968, pp. 517-524.

[24] G. Allasia, Simultaneous interpolation and approximation by a class of multivariate positive operators, Numerical Algorithms 34 (2-4) (2003) $147-158$.

[25] D. Thilakawardana, K. Moessner, A genetic approach to cell-by-cell dynamic spectrum allocation for optimising spectral efficiency in wireless mobile systems, in: Cognitive Radio Oriented Wireless Networks and Communications, 2007. CrownCom 2007. 2nd International Conference on, 2007, pp. 367-372. doi:10.1109/CROWNCOM.2007.4549825.

[26] S. B. et al, Federating wired and wireless test facilities through emulab and omf: the ilab.t use case" in the proceedings of tridentcom 2012, in: Proceedings of TridentCom, 2012.

[27] A. Gamal, J. Mammen, B. Prabhakar, D. Shah, Throughput-delay trade-off in wireless networks, in: INFOCOM 2004. Twenty-third AnnualJoint Conference of the IEEE Computer and Communications Societies, Vol. 1, 2004, pp. -475 . doi:10.1109/INFCOM.2004.1354518.

[28] ITU-T recommendation G.107. International telephone connection and circuits. General definitions. The E model, a computational model for use in transmission planning (Mar. 2005).
[29] Iperf - the TCP/UDP bandwidth measurement tool, http://iperf.fr/, [Online; accessed 2013-07-19].

[30] D. Plets, M. Pakparvar, W. Joseph, L. Martens, Influence of intra-network interference on quality of service in wireless lans, in: IEEE International Symposium on Broadband Multimedia Systems and Broadcasting (BMSB'13), London, UK, 2013.

[31] P. Singh, D. Deschrijver, T. Dhaene, A balanced sequential design strategy for global surrogate modeling, in: Winter Simulation Conference (Wintersim 2013), Washington DC (USA). 